\title{
Sensitivity-based approaches for handling discrete variables in optimal power flow computations
}

\author{
Florin Capitanescu and Louis Wehenkel, Member, IEEE
}

\begin{abstract}
This paper proposes and compares three iterative approaches for handling discrete variables in optimal power flow (OPF) computations. The first two approaches rely on the sensitivities of the objective and inequality constraints with respect to discrete variables. They set the discrete variables values either by solving a mixed integer linear programming (MILP) problem or by using a simple procedure based on a merit function. The third approach relies on the use of Lagrange multipliers corresponding to the discrete variables bound constraints at the OPF solution. The classical round-off technique and a progressive round-off approach have been also used as a basis of comparison. We provide extensive numerical results with these approaches on four test systems with up to 1203 buses, and for two OPF problems: loss minimization and generation cost minimization, respectively. These results show that the sensitivity-based approach combined with the merit function clearly outperforms the other approaches in terms of: objective function quality, reliability, and computational times. Furthermore, the objective value obtained with this approach has been very close to that provided by the continuous relaxation OPF. This approach constitutes therefore a viable alternative to other methods dealing with discrete variables in an OPF.
\end{abstract}

Index Terms-discrete variables, mixed integer linear programming, mixed integer nonlinear programming, nonlinear programming, optimal power flow

\section{INTRODUCTION}

$\mathbf{T}$ HE Optimal Power Flow (OPF) problem [1], [2] is a non-convex, large-scale, nonlinear programming problem with both continuous and discrete variables, in brief a mixedinteger nonlinear programming (MINLP) problem.

The efficient handling of discrete variables in the OPF has been recognized as a challenging problem and has received significant attention since the late 80's [3]-[6]. Since then a large spectrum of heuristic approaches have been proposed to deal with discrete variables e.g. round-off techniques [4], [5], [7], methods handling discrete variables in NLP and LP OPF formulations by means of penalty functions [8]-[10], ordinal optimization [11], recursive mixed-integer linear programming [12], interior point cutting plane [13], [14], global optimization methods [15]-[18], etc.

The simplest approach for handling discrete variables is based on the round-off strategy [4], [5]. In this technique, the OPF relaxation is first solved by treating all variables as continuous. Next, at the optimal solution, the discrete variables are rounded-off to their nearest discrete value. Finally, the discrete variables are frozen and the continuous variables are determined by re-solving the OPF. To reduce the sharp effect

The authors are with the Department of Electrical Engineering and Computer Science, University of Liège, B4000 Liège, Belgium (e-mail: capitane@montefiore.ulg.ac.be; 1.wehenkel@ulg.ac.be). of rounding-off all discrete variables at once, a progressive rounding-off of discrete variables has been proposed [7]. In this approach at each iteration only a subset of discrete variables which are sufficiently close to a discrete value are rounded-off, the remaining variables (treated as continuous) being then re-optimized. However, the round-off approaches act "blindly" since they do not look at the discretization effect on the objective or the constraints, suffering in consequence from two drawbacks: (a) no back-up solution is proposed if the chosen discrete variables values lead to an infeasible OPF problem for the continuous variables, and (b) it may lead to poor sub-optimal solutions of the OPF problem.

The treatment of discrete variables by means of penalty terms appended to the OPF objective has been proposed in the context of the active-set Newton OPF method [8], and the interior point method (IPM) [9]. The former approach uses several complicated heuristic rules to drive the discrete variables to discrete values. The latter approach provides results comparable with the performances of IPM solution of OPF relaxation. However, it uses very small steps for shunt banks (e.g. maximum 6 Mvar), whereas in real-life these steps are often significantly larger (e.g. up to 30-40 Mvar). Our experience with this approach shows that its convergence may be prohibitively slow when considering larger steps, and that even sometimes it does not converge at all.

An approach using ordinal optimization theory is proposed in [11]. It uses first a continuous OPF computation. Then it reduces the search space of discrete variables by keeping only the two closest discrete values to the continuous solution. Finally, the OPF is solved only for some combinations of discrete values, selected by means of ordinal optimization theory according to their estimated impact on the objective.

Last but not least the OPF problem has received a particular attention from the perspective of global optimization methods, e.g. genetic algorithms [15], simulated annealing [16], tabu search [17], or hybrid approaches coupling genetic algorithms and local search NLP solvers [18]. However, these techniques still remain to be evaluated on large scale applications in terms of computational complexity and robustness.

In this paper, we propose a heuristic approach able to cope with discrete variables in large scale OPF computations and suitable for near real-time applications. In a nutshell, this approach works as follows:

- first, the discrete variables (shunt compensation, transformer taps, etc.) are treated as continuous and a relaxed solution is computed by a classical OPF;

- next, an iterative procedure is applied, which at each iteration moves a subset of discrete variables to their 
closest upper or lower discrete position and then uses an OPF to re-optimize the continuous variables only;

- the iterative procedure is terminated once a feasible solution is found whose integrality gap is sufficiently small or when no significant improvement of the objective is observed with respect to the previous iteration.

The remaining of the paper is organized as follows. Section II recalls the general OPF formulation. Sections III and IV present the proposed approaches, respectively based on sensitivities and Lagrange multipliers. Section V offers detailed numerical results. We provide our conclusions in Section VI.

\section{The Optimal Power Flow Problem}

The classical OPF problem can be written as follows:

$$
\begin{array}{rl}
\min _{\mathbf{x}, \mathbf{c}, \mathbf{d}} & f(\mathbf{x}, \mathbf{c}, \mathbf{d}) \\
\text { s.t. } & \mathbf{g}(\mathbf{x}, \mathbf{c}, \mathbf{d})=\mathbf{0} \\
\mathbf{h}(\mathbf{x}, \mathbf{c}, \mathbf{d}) \leq \mathbf{0} \\
\quad \underline{\mathbf{c}} \leq \mathbf{c} \leq \overline{\mathbf{c}} \\
\mathbf{d}=\left[d_{1} \ldots d_{i} \ldots d_{n_{d}}\right]^{T} \\
\quad d_{i} \in\left\{d_{i}^{1}, \ldots, d_{i}^{j}, \ldots, d_{i}^{p(i)}\right\} \forall i=1, \ldots, n_{d},
\end{array}
$$

where $\mathbf{x}$ is the vector of state variables (i.e. real and imaginary part of voltage at all buses), $\mathbf{c}$ is an $n_{c}$ dimensional vector of continuous control variables (e.g. generators active power, generators voltage, etc.) and $\underline{\mathbf{c}}$ (resp. $\overline{\mathbf{c}}$ ) is its corresponding vector of lower (resp. upper) bounds, $\mathbf{d}$ is an $n_{d}$ dimensional vector of discrete control variables (e.g. load tap changer (LTC) transformer ratios, shunt element reactances, phase shifters angle, etc.), $d_{i}^{j}$ is the $j$-th discrete value of discrete variable $d_{i}, p(i)$ is the number of discrete positions of discrete variable $d_{i}, f(\cdot)$ is the objective function, $\mathbf{g}(\cdot)$ and $\mathbf{h}(\cdot)$ are vectors of functions which model equality and inequality constraints. Equality constraints (2) are essentially the ac bus power flow equations, inequality constraints (3) refer to operational limits (e.g. branch currents and voltage magnitudes), inequality constraints (4) refer to physical limits of equipments (e.g. bounds on: generators active/reactive powers, etc.), and constraints (5) describe the discrete variable values.

A configuration of the discrete variables $\mathbf{d}^{s}$ is called feasible if the OPF problem (1-4) in which the constraint $\mathbf{d}=\mathbf{d}^{s}$ is imposed is feasible. Otherwise, it is called infeasible.

Notice that an exhaustive enumeration approach applied to the OPF problem (1-5) would require the consideration of $\prod_{i=1}^{n_{d}} p(i)$ configurations. To fix ideas, consider a medium sized OPF problem with say $n_{d}=100$ discrete variables corresponding to 100 transformers with $p=20$ steps. In this case the number of possible configurations would be of $20^{100}$ which is intractable by an enumeration approach.

\section{Setting Discrete VARiables by SEnSitivities}

\section{A. The underlying idea of the proposed approach}

The underlying idea of the proposed approach is to set values of discrete variables by assessing their ability to improve the objective and to remove inequality constraint violations. The benchmark approach to fulfill this task is based on finite differences. In this approach each discrete variable is moved to a new discrete position, a power flow (PF) computation is performed, and at its solution the variation of the objective and inequality constraints due to variable change is computed. This approach is however very time consuming and does not ensure that the simultaneous changes in several discrete variables will be modeled with sufficient accuracy by superposing their individual effects.

In order to speed up computations we use instead an approach based on first order analytical sensitivities. Furthermore, since the validity of sensitivities is ensured only for small variations around the operating point where they are derived, we restrict the discrete variables changes to a single step (up or down) per iteration.

\section{B. Derivation of sensitivities with respect to discrete variables}

Let $(\hat{\mathbf{x}}, \hat{\mathbf{c}}, \hat{\mathbf{d}})$ be a proposal solution stemming from an OPF or a PF computation, and denote by $j_{i}\left(\forall i=1, \ldots, n_{d}\right)$ the current position of discrete variable $d_{i}$.

The key information of our approach is the sensitivity of the objective function and inequality constraints with respect to discrete variables $\mathbf{d}$ changes, which we denote by $\mathbf{S}_{\mathbf{d}}^{f}$ and $\mathbf{S}_{\mathbf{d}}^{\mathbf{h}}$ respectively. In the literature there exist analytical formulas to compute these sensitivities for an equilibrium point of the system (e.g. the solution of an OPF or a PF) [2], [19]. According to [2] these sensitivities take on the form:

$$
\begin{gathered}
\mathbf{S}_{\mathbf{d}}^{f}=\frac{\partial f}{\partial \mathbf{d}}-\left(\frac{\partial \mathbf{g}}{\partial \mathbf{d}}\right)^{T}\left[\left(\frac{\partial \mathbf{g}}{\partial \mathbf{x}}\right)^{T}\right]^{-1} \frac{\partial f}{\partial \mathbf{x}} \\
\mathbf{S}_{\mathbf{d}}^{\mathbf{h}}=\frac{\partial \mathbf{h}}{\partial \mathbf{d}}-\frac{\partial \mathbf{h}}{\partial \mathbf{x}}\left(\frac{\partial \mathbf{g}}{\partial \mathbf{x}}\right)^{-1} \frac{\partial \mathbf{g}}{\partial \mathbf{d}}
\end{gathered}
$$

We note that, if the point where the sensitivities are derived is an OPF optimum, the terms $\left[\left(\frac{\partial \mathbf{g}}{\partial \mathbf{x}}\right)^{T}\right]^{-1} \frac{\partial f}{\partial \mathbf{x}}$ and $\frac{\partial \mathbf{h}}{\partial \mathbf{x}}\left(\frac{\partial \mathbf{g}}{\partial \mathbf{x}}\right)^{-1}$ involved in (6) and (7) are the vectors of Lagrange multipliers of equality constraints (2) and inequality constraints (3); hence they are provided as a by-product of the OPF computation [2]. While the former term is very useful and can be used directly in (6) to speed up sensitivities computation, the latter term is less useful since only those components that correspond to binding inequality constraints are non-zero. Also the incremental computational effort to derive these sensitivities is very small, since the factorized power flow Jacobian $\frac{\partial \mathbf{g}}{\partial \mathbf{x}}$ is a by-product at the solution of an OPF or a PF program.

\section{Setting discrete variables values by MILP}

Starting from an initial configuration of the discrete variables $d_{i}^{j_{i}}, \forall i$, a new configuration may be computed by solving the following MILP problem, which relies on the sensitivities of the objective and the inequality constraints violations with respect to discrete variables (6) and (7):

$$
\begin{aligned}
& \min _{\mathbf{d}} \sum_{i=1}^{n_{d}} S_{d_{i}}^{f}\left(d_{i}-d_{i}^{j_{i}}\right) \\
& \text { s.t. } h_{k}(\hat{\mathbf{x}}, \hat{\mathbf{c}}, \hat{\mathbf{d}})+\sum_{i=1}^{n_{d}} S_{d_{i}}^{h_{k}}\left(d_{i}-d_{i}^{j_{i}}\right) \leq 0 \quad k=1, \ldots, n_{h} \\
& \qquad d_{i} \in\left\{d_{i}^{j_{i}-1}, d_{i}^{j_{i}}, d_{i}^{j_{i}+1}\right\} i=1, \ldots, n_{d}
\end{aligned}
$$


where, for each discrete variable $d_{i}^{j_{i}-1}$ (resp. $d_{i}^{j_{i}+1}$ ) is its nearest lower (resp. higher) discrete value with respect to the current value, and $n_{h}$ is the dimension of vector $\mathbf{h}$ in (3).

Observe that the objective function of the MILP problem is the linearized original objective function and that its inequality constraints (9) are the linearizations of the original OPF constraints (3). Also, the discrete constraints (10) impose that each discrete variable can take one of three successive values, namely its current value or the closest upper or lower value. Note that if the initial configuration of a discrete variable is not in the discrete set (i.e. $d_{i} \notin\left\{d_{i}^{1}, \ldots, d_{i}^{j}, \ldots, d_{i}^{p(i)}\right\}$ ), e.g. due to a relaxed OPF computation, we write equation (10) as $d_{i} \in\left\{d_{i}^{j_{i}}, d_{i}^{j_{i}+1}\right\}$, where $d_{i}^{j_{i}}$ and $d_{i}^{j_{i}+1}$ are respectively the closest lower and upper discrete values with respect to the continuous value of variable $d_{i}$.

To solve the above MILP problem we use the GLPK-v4.35 solver [20] which uses a branch and cut algorithm.

\section{Iterative MILP based algorithm}

The proposed iterative algorithm is outlined as follows:

1) Solve the OPF continuous relaxation (discrete variables are treated as continuous).

2) At the current OPF optimum, compute the sensitivity of the objective function and inequality constraints with respect to discrete variables $\left(\mathbf{S}_{\mathbf{d}}^{f}\right.$ and $\left.\mathbf{S}_{\mathbf{d}}^{\mathbf{h}}\right)$ according to formulas (6) and (7).

3) Solve the MILP problem (8-10) in order to set the new discrete values of discrete variables. Let $\mathbf{d}^{\star}$ be solution of the problem.

4) Solve the OPF to optimize continuous variables only (discrete variables are frozen at their current value $\mathbf{d}^{\star}$ ).

- If the OPF objective value change with respect to the previous iteration is smaller than a tolerance, then the current discrete solution can not be further improved significantly and the computation terminates. Besides, if the OPF objective is very close to that of the continuous relaxation OPF, then a nearoptimal solution has been found.

- Otherwise, go to step 2.

We observe that, unlike most methods existing in the literature, which search in one single shot for the optimal value of discrete variables by considering only two discrete values for each one of them, the proposed approach allows the iterative movement of discrete variables (one single step at the time) to positions that best improve the optimality while ensuring feasibility. In other words, the proposed approach uses a larger discrete search space and hence has higher chances to find a near-optimal feasible solution.

Although we formulate the algorithm by assuming that it searches from scratch, one may adapt it in order to take advantage of any other suitable approach for choosing an initial value of discrete variables (e.g. stemming from a realtime operating point or determined by the round-off approach choosing the closest discrete values to a relaxed OPF solution).

Note that the MILP problem solved at step 3) of the algorithm may be infeasible due to slight sensitivities inaccuracy or due to overly limited range of discrete controls. We deal with MILP infeasibility by progressively relaxing constraints until the problem becomes feasible.

Finally, it may also happen that the OPF problem posed at step 2) is infeasible (e.g. due to the choice of discrete variables at a given stage of the procedure). To lighten the algorithm comprehension this case is discussed in Section III-F.

\section{E. Setting discrete variables values by merit functions}

The underlying idea of the merit function based approach is to set values of discrete variables in a greedy fashion by assessing their individual ability to improve the objective and to remove inequality constraints violations.

For each discrete variable $d_{i}\left(\forall i=1, \ldots, n_{d}\right)$, one can indeed estimate linearly the change in the objective and the inequality constraints when moving $d_{i}$ from its current value $d_{i}^{j_{i}}$ to its nearest upper or lower value by

$$
\begin{aligned}
\Delta f_{i}^{+} & =S_{d_{i}}^{f}\left(d_{i}^{j_{i}+1}-d_{i}^{j_{i}}\right) \\
\Delta f_{i}^{-} & =S_{d_{i}}^{f}\left(d_{i}^{j_{i}-1}-d_{i}^{j_{i}}\right) \\
\Delta h_{k i}^{+} & =S_{d_{i}}^{h_{k}}\left(d_{i}^{j_{i}+1}-d_{i}^{j_{i}}\right),
\end{aligned}
$$

From this we may compute the value of two merit functions corresponding to the movement of $d_{i}$ to its nearest upper and lower discrete value by combining the variation of the objective and the variation of the amount of constraints violation:

$$
\begin{gathered}
\eta_{i}^{+}=w_{f} \Delta f_{i}^{+}+\sum_{k=1}^{n_{h}} w_{h} \max \left[0, h_{k}(\hat{\mathbf{x}}, \hat{\mathbf{c}}, \hat{\mathbf{d}})+\Delta h_{k i}^{+}\right] \\
\eta_{i}^{-}=w_{f} \Delta f_{i}^{-}+\sum_{k=1}^{n_{h}} w_{h} \max \left[0, h_{k}(\hat{\mathbf{x}}, \hat{\mathbf{c}}, \hat{\mathbf{d}})+\Delta h_{k i}^{-}\right],
\end{gathered}
$$

where $w_{f} \geq 0$ and $w_{h} \geq 0$ are weighting factors.

Observe that the terms related to inequality constraints contribute to the merit function only if some inequality constraints are violated after altering the value of discrete variable $d_{i}$, i.e. if $h_{k}(\hat{\mathbf{x}}, \hat{\mathbf{c}}, \hat{\mathbf{d}})+\Delta h_{k i}^{+}>0$ or $h_{k}(\hat{\mathbf{x}}, \hat{\mathbf{c}}, \hat{\mathbf{d}})+\Delta h_{k i}^{-}>0$.

Clearly, the lower the value of the merit function the better the effect of moving a discrete variable. Furthermore, a discrete variable is actually modified only if its effect is deemed significant i.e. its merit function is lower than threshold $\eta_{t h}$. The value of $\eta_{t h}$ can be adapted in order to control the number of discrete variables allowed to move at a given iteration.

Finally, the discrete variable $d_{i}$ is moved to its nearest discrete value which leads to the least value among both merit functions provided that this value is lower than $\eta_{t h}$, i.e.:

$$
\begin{array}{lll}
d_{i}^{j_{i}} \leftarrow d_{i}^{j_{i}+1} & \text { if: } & \eta_{i}^{+} \leq \eta_{i}^{-} \text {and } \eta_{i}^{+} \leq \eta_{t h} \text { or } \\
d_{i}^{j_{i}} \leftarrow d_{i}^{j_{i}-1} & \text { if: } \quad \eta_{i}^{-}<\eta_{i}^{+} \text {and } \eta_{i}^{-} \leq \eta_{t h}
\end{array}
$$

Notice that in the above procedure we assume that the discrete variables at the current iteration are already at discrete values. However, depending on the procedure used to set discrete variables (e.g. starting with a continuous relaxation of the OPF) this is generally not the case. In the situation where variable $d_{i}^{j_{i}}$ does not have a discrete value, $d_{i}^{j_{i}+1}$ and $d_{i}^{j_{i}-1}$ from formulas (11-14) represent its nearest upper and lower discrete values while $\eta_{t h} \rightarrow \infty$ in (17-18). 
In order to take explicitly into account the potential cumulated effects of discrete variables on inequality constraints (e.g. if discrete variables are related to devices which are located close to each other in the power system) we use a simple heuristic to update their values:

1) rank the variables by increasing order of merit;

2) pick the top ranked one which has not been set yet to a discrete value and set it to its best discrete value;

3) update the inequality constraints (e.g. the bus voltage magnitudes) by considering linearly the effect of changing the value of the top ranked discrete variable;

4) if all discrete variables have been set to a discrete value stop, otherwise go to step 1.

\section{F. Dealing with continuous OPF infeasibility}

As mentioned above, it is possible that during the iterative search of satisfactory configurations of discrete variables some of the configurations proposed by our algorithm lead to an OPF problem (1-4) which is infeasible. To the authors' knowledge no approach proposed in the literature integrates techniques to deal with infeasible configurations of discrete variables.

To handle such situations we propose to solve a minimum amount of constraints violation OPF problem instead of the original problem. Thus, if for a configuration of the discrete variables the OPF (1-4) with continuous variables only is infeasible, we solve instead the following problem:

$$
\begin{aligned}
& \min _{\mathbf{x}, \mathbf{c}, \mathbf{r}} \mathbf{1}^{T} \mathbf{r} \\
& \text { s.t. } \mathbf{g}(\mathbf{x}, \mathbf{c}, \mathbf{d})=\mathbf{0} \\
& \mathbf{h}(\mathbf{x}, \mathbf{c}, \mathbf{d}) \leq \mathbf{r} \\
& \mathbf{c} \leq \mathbf{c} \leq \mathbf{\mathbf { c }} \\
& \mathbf{r} \geq \mathbf{0},
\end{aligned}
$$

in which positive relaxation variables (23) have been introduced in order to relax the inequality constraints (21). The objective (19) is the minimization of the amount of constraints violation in the sense of the $L_{1}$ norm.

The solution of problem (19-23) in the context of the proposed algorithm is very useful since it provides an optimum of the relaxed OPF which enables the derivation of sensitivities (6-7) and hence allows to carry on the algorithm. Incidentally, the solution of the problem (19-23) yields the amount of original continuous OPF problem infeasibility. This is a worthy information especially if the MINLP OPF (1-5) is infeasible whatever the values taken by discrete variables.

\section{Setting Discrete VARIABLES VALUES FROM LAGRANGE MULTIPLIERS AT THE OPF SOLUTION}

The use of Lagrange multipliers derived by an OPF program is another approach to set discrete variable values which, unlike the approach of Section III, does not require to explicitly take care of the impact of discrete variables changes on the violated inequality constraints. Indeed, exploiting Lagrange multipliers at the OPF solution relies on the observation that if a discrete variable is binding at the OPF solution, then its corresponding Lagrange multiplier indicates the sensitivity of the objective to a small variation of this discrete variable along all directions respecting the constraints. The underlying assumption of this approach is that the binding constraints of the OPF do not change significantly following discrete variable changes. This assumption holds generally true for very small variations of discrete variables around the OPF solution. However, for a large excursion of a discrete variable and/or when many discrete controls vary simultaneously this assumption may not hold true anymore.

We propose the following algorithm to exploit the Lagrange multipliers at the OPF solution:

1) Assume a given feasible configuration for discrete variables $d_{i}=d_{i}^{j_{i}}\left(\forall i=1, \ldots, n_{d}\right)$ (e.g. provided by the round-off approach). Let $\Delta f_{\min }$ be the minimum required improvement of the objective function.

2) Solve the OPF so as to optimize continuous variables while keeping frozen discrete variables, i.e. $d_{i}=d_{i}^{j_{i}}$, $\forall i=1, \ldots, n_{d}$. If the OPF objective value change with respect to the previous iteration is smaller than a tolerance, then the current discrete solution can not be further improved significantly and the computation terminates. Besides, if the OPF objective is very close to that of the continuous relaxation OPF, then a nearoptimal solution has been found.

3) Solve a modified OPF by considering discrete variables as continuous in a small range: $d_{i} \in\left[d_{i}^{j_{i}}-\varepsilon d_{i}^{j_{i}}+\varepsilon\right]$. At the OPF solution let $m_{i 1}$ (resp. $m_{i 2}$ ) be the Lagrange multiplier corresponding to constraint $d_{i} \geq d_{i}^{j_{i}}-\varepsilon$ (resp. $\left.d_{i} \leq d_{i}^{j_{i}}+\varepsilon\right)$.

4) Update discrete variables values, i.e. $\forall i$ do:

- if $m_{i 1}>0$ and $m_{i 1}\left(d_{i}^{j_{i}}-d_{i}^{j_{i}-1}\right) \geq \Delta f_{\min }$ then $d_{i}^{j_{i}} \leftarrow d_{i}^{j_{i}-1}$.

- else, if $m_{i 2}>0$ and $m_{i 2}\left(d_{i}^{j_{i}+1}-d_{i}^{j_{i}}\right) \geq \Delta f_{\min }$ then $d_{i}^{j_{i}} \leftarrow d_{i}^{j_{i}+1}$.

5) If at step (4) none of the discrete variables has changed its position then an acceptable solution has been found and the computation terminates. Otherwise, go to step 2.

The main information in the above algorithm is brought by the values of Lagrange multipliers $m_{i 1}$ and $m_{i 2}$. Thus, for each discrete variable $d_{i}, m_{i 1}>0$ means that decreasing its value slightly leads to a larger improvement of the objective than increasing it. Also a discrete variable remains frozen if its movement to the next upper or lower position would not impact significantly the objective value.

\section{NumERICAL CASE STUdies}

\section{A. Test systems and OPF problems description}

We present detailed results obtained with several approaches in four test systems: a 60-bus system [21], which is a modified variant of the Nordic32 system [22], the IEEE300 bus system [21], [23], a 618-bus system which is a modified old planning model of a region of the RTE (the French transmission system operator) system, and a 1203-bus system which is a modified ${ }^{1}$ old planning model of the whole RTE system of 1203 buses.

\footnotetext{
${ }^{1}$ for the sake of dealing with a large number of discrete variables we have significantly increased the number of LTC transformers and shunt banks
} 
TABLE I

TEST SYSTEMS SUMMARY

\begin{tabular}{|c||c|c|c|c|c|c|c|c||c|c|}
\hline system & $n$ & $g$ & $c$ & $b$ & $l$ & $t$ & $o$ & $s$ & $n_{c}$ & $n_{d}$ \\
\hline \hline Nordic32 & 60 & 23 & 22 & 81 & 57 & 31 & 4 & 12 & 24 & 16 \\
\hline IEEE300 & 300 & 69 & 198 & 411 & 282 & 129 & 50 & 14 & 70 & 54 \\
\hline 618-bus & 618 & 72 & 352 & 1057 & 810 & 247 & 175 & 25 & 73 & 200 \\
\hline 1203-bus & 1203 & 177 & 767 & 1797 & 1394 & 403 & 203 & 36 & 178 & 239 \\
\hline
\end{tabular}

A summary of these systems characteristics is given in Table I. In this table $n, g, c, b, l, t, o, s, n_{c}$, and $n_{d}$ denote respectively the number of: buses, generators, loads, branches, lines, all transformers, transformers with controllable ratio, shunt elements, continuous control variables, and discrete control variables. All tests have been performed on a PC 1.7$\mathrm{GHz}$ Pentium IV with 512-Mb RAM. In all our trials we use the interior point OPF solver described in [24].

The OPF data of the 60-bus and IEEE300-bus systems used in this paper, as well as the relevant numerical results obtained with the various approaches under study, have been archived and made publicly available for comparison purposes [21].

We provide extensive numerical results for two OPF problems: the minimization of active power losses and the minimization of the generation cost.

We formulate the OPF problem (1-5) with voltages expressed in rectangular coordinates:

$$
\underline{V}_{i}=e_{i}+j f_{i}, \quad i=1, \ldots, n .
$$

The OPF objective function (1) is either the minimum active power losses:

$$
\min \sum_{i=1}^{n} \sum_{j=1}^{n} G_{i j}\left[\left(e_{i}-e_{j}\right)^{2}+\left(f_{i}-f_{j}\right)^{2}\right],
$$

or the minimum generation cost:

$$
\min \sum_{i=1}^{g}\left(c_{0 i}+c_{1 i} P_{G i}+c_{2 i} P_{G i}^{2}\right),
$$

where, the cost curve of generator $i$ is defined by coefficients $c_{0 i}, c_{1 i}$, and $c_{2 i}$, while $P_{G i}$ is the generator output.

For the minimum power losses OPF problem we consider both continuous (e.g. generator terminal voltages) and discrete control variables (e.g. transformer ratios and shunt reactances), while for the OPF problem of generation cost minimization we additionally use generators' active powers as control variable.

OPF constraints (2) encompass the active and reactive power flow equations at each bus $i(i=1, \ldots, n)$ :

$$
\begin{gathered}
P_{G i}-P_{L i}-\left(e_{i}^{2}+f_{i}^{2}\right) \sum_{j \in N_{i}}\left(G_{s i j}+G_{i j}\right) \\
+\sum_{j \in N_{i}}\left[\left(e_{i} e_{j}+f_{i} f_{j}\right) G_{i j}+\left(f_{i} e_{j}-e_{i} f_{j}\right) B_{i j}\right]=0, \\
Q_{G i}-Q_{L i}+\left(e_{i}^{2}+f_{i}^{2}\right)\left[B_{s i}+\sum_{j \in N_{i}}\left(B_{s i j}+B_{i j}\right)\right] \\
-\sum_{j \in N_{i}}\left[\left(e_{i} e_{j}+f_{i} f_{j}\right) B_{i j}+\left(e_{i} f_{j}-f_{i} e_{j}\right) G_{i j}\right]=0,
\end{gathered}
$$

where $N_{i}$ is the set of neighbor buses of bus $i$, other notations being self-explanatory.
OPF constraints (3) include operational limits on branch currents and voltage magnitudes:

$$
\begin{array}{r}
\left(G_{i j}^{2}+B_{i j}^{2}\right)\left[\left(e_{i}-e_{j}\right)^{2}+\left(f_{i}-f_{j}\right)^{2}\right] \leq\left(I_{i j}^{\max }\right)^{2} \\
\left(V_{i}^{\min }\right)^{2} \leq e_{i}^{2}+f_{i}^{2} \leq\left(V_{i}^{\max }\right)^{2} \\
i=1, \ldots, n, \quad j \in N_{i}
\end{array}
$$

We use voltage limits of $0.95 \mathrm{pu}$ and $1.05 \mathrm{pu}$ at all buses.

OPF constraints (4) include physical limits of power system devices:

$$
\begin{aligned}
P_{G i}^{\min } & \leq P_{G i} \leq P_{G i}^{\max }, i=1, \ldots, g \\
Q_{G i}^{\min } & \leq Q_{G i} \leq Q_{G i}^{\max }, i=1, \ldots, g
\end{aligned}
$$

Finally, OPF constraints (5) model discrete variables:

$$
\begin{gathered}
r_{i} \in\left\{r_{i}^{\min }, r_{i}^{\min }+s_{r_{i}}, r_{i}^{\min }+2 s_{r_{i}}, \ldots, r_{i}^{\max }\right\}, i=1, \ldots, o \\
b_{i} \in\left\{b_{i}^{\min }, b_{i}^{\min }+s_{b_{i}}, b_{i}^{\min }+2 s_{b_{i}}, \ldots, b_{i}^{\max }\right\}, i=1, \ldots, s,
\end{gathered}
$$

where $s_{r_{i}}$ is the ratio step of transformer $i$ and $s_{b_{i}}$ is the susceptance step of shunt $i$.

We assume that transformer ratios may vary between $0.9 \mathrm{pu}$ and $1.1 \mathrm{pu}$ with a step of $0.01 \mathrm{pu}$ ( 21 positions), while steps of the shunt compensation banks range from 8 to 40 Mvar (see the set B of discrete controls step sizes in Table VII).

\section{B. Analytical sensitivities validation by finite differences}

Table II shows the objective function change obtained by moving one discrete control at the time as determined by two approaches: finite differences based on a power flow computation and predictions using our analytical sensitivities. The approach by finite differences shows the objective shift when moving each control with one discrete step either up or down. The step for any LTC ratio is of $0.01 \mathrm{pu}$ and for any shunt of 30 MVar. For this comparison we use a non-optimized base case (e.g. a power flow solution) of the 1203-bus system.

We observe from Table II that the objective function change computed by both approaches is generally rather close. Clearly, small mismatches are expected due to the well known nonlinear local effect of reactive power flow. Observe also that for some controls (e.g. LTC 191 and shunt 34) increasing and decreasing with the same amount the current value of a discrete variable leads to rather different values. This mismatch is also owing to the non-linearity introduced by generators which lose or regain their ability to control voltage subsequently to the action of other "reactive" power control devices (e.g. LTC ratio, shunt reactance, etc.). For instance, at the base case 15 generators have reached their maximum reactive power limit (these constraints are binding at the continuous OPF solution) but when injecting some reactive power into the network, all these generators come back under voltage control.

Figs. 1 and 2 plot the variation of active power losses respectively when moving the taps of two transformers or when changing two shunts compensators. We have considered for both a discrete variation of 5 steps up or down with respect to the base case. These figures clearly show that, except for some controls, analytical sensitivities cannot be trusted for variations of the current value larger than one discrete step, 
TABLE II

OBJECTIVE FUNCTION CHANGE COMPUTED BY FINITE DIFFERENCES AND PREDICTED BY ANALYTICAL SENSITIVITIES FOR VARIOUS DISCRETE CONTROL CHANGES (ONE CONTROL VARIABLE CHANGE AT THE TIME): RESULTS USING THE 1203-BUS SYSTEM

\begin{tabular}{|c|c|c|c|c|c|c|c|c|c|}
\hline \multicolumn{8}{|c|}{ LTC number } & \multicolumn{6}{c|}{ shunt number } \\
\hline 3 & 10 & 83 & 132 & 191 & 3 & 6 & 17 & 21 & 34 \\
\hline \multicolumn{8}{|c|}{ real objective change (MW) by finite differences } \\
\hline 0.16 & 0.18 & -0.02 & 0.17 & 0.31 & -0.23 & -0.46 & -0.72 & -0.84 & -0.32 \\
-0.12 & -0.16 & 0.04 & -0.15 & -0.23 & 0.24 & 0.53 & 0.75 & 0.92 & 0.45 \\
\hline \multicolumn{8}{|c|}{ predicted objective change (MW) by analytical sensitivities } \\
\hline \hline 0.16 & 0.20 & -0.06 & 0.18 & 0.37 & -0.24 & -0.52 & -0.74 & -0.89 & -0.39 \\
\hline
\end{tabular}

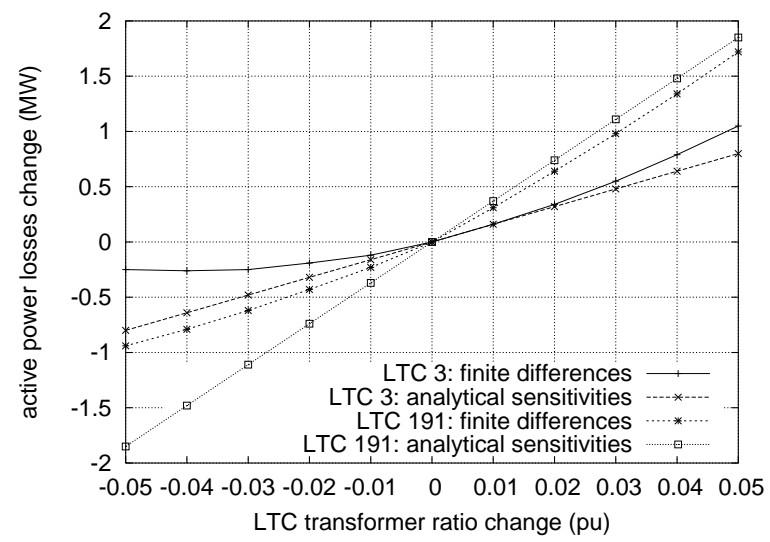

Fig. 1. The active power losses change with respect to transformer LTC ratio: finite differences vs. analytical sensitivities

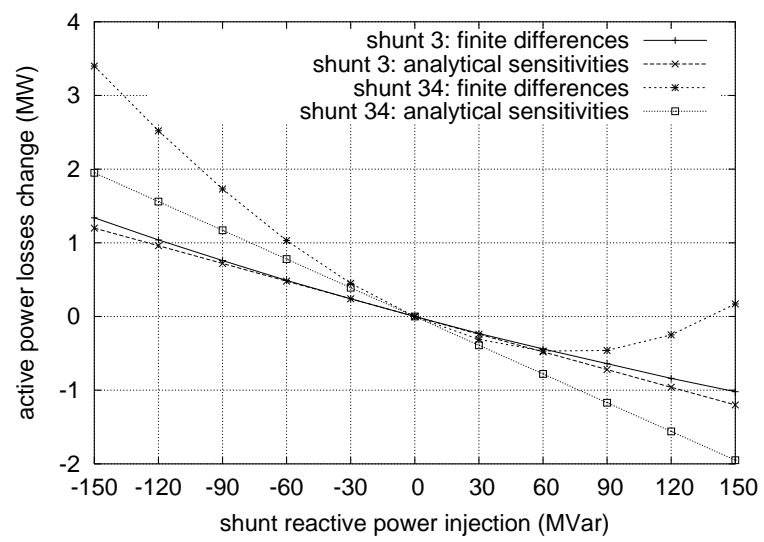

Fig. 2. The active power losses change with respect to shunt reactive power injection: finite differences vs. analytical sensitivities

which justifies our strategy to allow a discrete variable to move at most one step up or down.

Table III provides on the other hand, for different pairs of weights $w_{f}$ and $w_{h}$ involved in the merit function formulas (15) and (16), the objective function change and the overall bus voltage limit violations, obtained by using the modified settings of the discrete controls suggested by the merit function heuristic. Here the variations predicted by the sensitivity-based approach are linear approximations, while the actual effects are computed by a power flow solver and take into account possible non linear effects as well.

Let us observe that there is a very good agreement among the two approaches concerning the overall bus voltage limit violations. On the other hand, the sensitivity-based approach
TABLE III

OBJECTIVE FUNCTION AND OVERALL BUS VOLTAGE LIMIT VIOLATION CHANGES COMPUTED BY PF AND PREDICTED BY ANALYTICAL SENSITIVITIES FOR SIMULTANEOUS CONTROL MOVEMENTS DETERMINED BY USING VARIOUS VALUES OF WEIGHTS $w_{f}$ AND $w_{h}$ : RESULTS USING THE 1203-BUS SYSTEM

\begin{tabular}{|c|c|c|c|c|c|c|}
\hline$w_{f}>0$ & $w_{f}=1$ & $w_{f}=1$ & $w_{f}=1$ & $w_{f}=1$ & $w_{f}=1$ & $w_{f}=0$ \\
$w_{h}=0$ & $w_{h}=1$ & $w_{h}=10$ & $w_{h}=50$ & $w_{h}=100$ & $w_{h}=200$ & $w_{h}>0$ \\
\hline \hline \multicolumn{7}{|c|}{ real objective variation (MW) computed by PF } \\
\hline-19.78 & -19.77 & -17.08 & -13.93 & -10.01 & -7.24 & 0.47 \\
\hline \hline \multicolumn{7}{|c|}{ predicted objective variation (MW) by analytical sensitivities } \\
\hline-27.83 & -27.73 & -23.76 & -20.28 & -15.35 & -11.98 & 0.10 \\
\hline \hline \multicolumn{7}{|c|}{ real overall bus voltage limits violation (pu) computed by PF } \\
\hline 1.381 & 1.145 & 0.266 & 0.083 & 0.028 & 0.008 & 0.000 \\
\hline predicted overall bus voltage limits violation (pu) by analytical sensitivities \\
\hline 1.221 & 1.028 & 0.255 & 0.082 & 0.028 & 0.008 & 0.000 \\
\hline
\end{tabular}

TABLE IV

OBJECTIVE CHANGE COMPUTED BY FINITE DIFFERENCES AND PREDICTED BY LAGRANGE MULTIPLIERS FOR VARIOUS DISCRETE CONTROLS: RESULTS USING THE 1203-BUS SYSTEM

\begin{tabular}{|c|c|c|c|c|c|c|c|}
\hline \multicolumn{5}{|c|}{ LTC number } & \multicolumn{4}{c|}{ shunt number } \\
\hline 5 & 152 & 157 & 158 & 19 & 20 & 21 & 22 \\
\hline \hline \multicolumn{7}{|c|}{ real objective change (MW) by finite differences } \\
\hline 0.96 & 1.18 & 1.21 & 1.20 & 1.92 & 2.89 & 2.62 & 4.76 \\
-0.19 & -0.10 & -0.13 & -0.13 & -0.73 & -0.66 & -0.72 & -0.67 \\
\hline \hline \multicolumn{7}{|c|}{ predicted objective change (MW) by Lagrange multipliers } \\
\hline-0.79 & -1.06 & -1.06 & -1.05 & -1.40 & -1.88 & -2.16 & -1.58 \\
\hline
\end{tabular}

tends to overestimate the change of the objective function by about $40-50 \%$. Since the sensitivities have been validated when moving controls one at the time, this mismatch is due to the large number of discrete variables which move (around 200 in average). In these conditions the mismatch between sensitivity-based and finite differences approaches seems reasonable.

Note also that, at the power flow (PF) solution obtained after discrete variables changes, the larger the weight relative to the objective function, the larger the objective improvement but the higher the overall voltage limit violations.

\section{On Lagrange multipliers limited capability prediction}

We now compare the change in the objective by two approaches: finite differences and using Lagrange multipliers at the OPF solution. We consider the 1203-bus system and use as base case the optimal solution obtained with the round-off approach (i.e. 2054.15 MW, see Table V).

We have first checked the validity of Lagrange multipliers for very small variations of discrete variables value (i.e. $0.001 \mathrm{pu}$ for any LTC ratio of 1 MVar for any shunt, respectively) and observed that there is a very good agreement between both approaches. Then we have used the actual discrete steps of variables (i.e. 0.01 pu for any LTC ratio and 30 MVar for any shunt, respectively), the results obtained being presented in Table IV. The latter provides the objective change due to the movement of one discrete control at the time, obtained by two approaches: finite differences and that using the Lagrange multipliers at the OPF solution. The approach by finite differences shows the real objective variation when moving each control with one discrete step either up or down. 
TABLE V

POWER LOSSES (MW) AND CPU TIMES (IN SECONDS) BY 6 APPROACHES

\begin{tabular}{|c|c|c|c|c|c|c|}
\hline system & $\mathrm{c}-\mathrm{OPF}$ & $\mathrm{RO}$ & PRO & S-MF & S-MILP & L-OPF \\
\hline & $\begin{array}{l}\text { losses } \\
\text { time }\end{array}$ & $\begin{array}{l}\text { losses } \\
\text { time }\end{array}$ & $\begin{array}{l}\text { losses } \\
\text { time }\end{array}$ & $\begin{array}{l}\text { losses } \\
\text { time }\end{array}$ & $\begin{array}{l}\text { losses } \\
\text { time }\end{array}$ & $\begin{array}{l}\text { losses } \\
\text { time }\end{array}$ \\
\hline Nordic32 & $\begin{array}{c}137.79 \\
0.14\end{array}$ & $\begin{array}{c}137.92 \\
0.30\end{array}$ & $\begin{array}{c}137.92 \\
0.59\end{array}$ & $\begin{array}{c}137.82 \\
0.32\end{array}$ & $\begin{array}{c}137.81 \\
0.33\end{array}$ & $\begin{array}{c}138.02 \\
0.63\end{array}$ \\
\hline IEEE300 & $\begin{array}{c}386.60 \\
0.95\end{array}$ & fail & fail & $\begin{array}{c}388.06 \\
2.04\end{array}$ & $\begin{array}{c}388.49 \\
2.06\end{array}$ & fail \\
\hline 618-bus & $\begin{array}{c}844.25 \\
2.71\end{array}$ & $\begin{array}{c}846.46 \\
5.62\end{array}$ & $\begin{array}{c}846.31 \\
11.6\end{array}$ & $\begin{array}{c}845.07 \\
5.96\end{array}$ & $\begin{array}{c}845.41 * \\
45.7 *\end{array}$ & $\begin{array}{c}845.85 \\
11.5\end{array}$ \\
\hline 1203-bus & $\begin{array}{c}2050.58 \\
12.1\end{array}$ & $\begin{array}{c}2054.15 \\
24.4\end{array}$ & $\begin{array}{c}2053.30 \\
49.2\end{array}$ & $\begin{array}{c}2051.67 \\
25.3\end{array}$ & $\begin{array}{c}2053.47^{*} \\
97.8^{*}\end{array}$ & $\begin{array}{c}2053.57 \\
49.5\end{array}$ \\
\hline
\end{tabular}

We note that the prediction of the objective change by the Lagrange multipliers is rather poor, especially when decreasing discrete variable values, and in particular for LTC ratios. This large mismatch is due to the significant change in the active set (i.e. the binding constraints) when shifting a discrete variable value. Now by implementing the change of these eight discrete variables simultaneously according to the Lagrange multipliers values and re-optimizing the continuous variables we have noticed that the active power losses have actually increased by $0.03 \mathrm{MW}$ with respect to the base case. We attribute this behavior to the even stronger change of the active set under the movement of these eight discrete variables. We conclude that, although theoretically very appealing, the Lagrange multipliers-based approach is inappropriate to cope with discrete variables setting.

\section{Comparison of various approaches}

1) Minimization of active power losses: Table V provides the values of active power losses and computational time obtained by applying the following six different approaches:

- c-OPF: uses a continuous relaxation of original the OPF (all variables are treated as continuous) and its output is provided as a basis for assessing the integrality gap;

- round-off (RO): uses the classical round-off strategy at the solution of the OPF continuous relaxation;

- progressive round-off (PRO): at each iteration only a subset of discrete variables of a given $\operatorname{size}^{2}$ are roundedoff, the remaining variables (treated as continuous) being then re-optimized [7].

- S-MF: uses sensitivities with the merit function approach (see Section III-E);

- S-MILP: uses the sensitivities with the MILP approach (see Section III-D);

- L-OPF: uses the approach of Section IV based on Lagrange multipliers.

In Table $\mathrm{V}$ the superscript $*$ appearing in S-MILP column for the 618-bus and the 1203-bus systems indicates that the original MILP problem is infeasible. However, by very slightly relaxing (e.g. with $0.001 \mathrm{pu}$ ) the voltage limits at all buses the MILP becomes feasible and the so-obtained results are shown

\footnotetext{
${ }^{2}$ we assume that all discrete variables have to be moved at discrete positions in 3 iterations, meaning that, if initially all discrete variables take continuous values, we discretize up to $\left[n_{d} / 3\right]+1$ variables per iteration, in decreasing order of their distance to a discrete value
}

in this table. Another manner to avoid the original MILP infeasibility is by adding to the objective penalty functions relative to constraints violation.

Note first that the sensitivity based approaches outperform all other ones in terms of optimality since they lead to better objective value in all tests. S-MF provides generally slightly better results than S-MILP (except for the Nordic32 system where it is slightly inferior). L-OPF only slightly improves the objective with respect to the round-off approach, providing even worse results for the Nordic32 system, for reasons already mentioned in Section V-C. Finally, we notice that, despite being two times slower, the PRO technique only slightly improves the objective with respect to the RO scheme.

One can also observe that, even when the number of discrete controls is rather large (e.g. for the 618-bus and 1203-bus systems), the discrete optimum reached is quite close to that of the continuous approach. This effect, also pointed out in other works (e.g. Refs. [4], [5], [9]), is due to the rather small sensitivity of the objective function with respect to discrete control changes for these test cases. As a consequence, all approaches have reached convergence in all cases in a single iteration of the algorithm (see Sections III-D and IV).

The S-MF approach appears also to be the most reliable since it always leads to feasible solutions. On the contrary, both RO and PRO techniques fail for the IEEE300 system (and so the L-OPF approach, since it is initialized by the round-off approach) because it leads to an infeasible continuous OPF after the round-off of discrete variables.

As regards computational times, the round-off approach is obviously the fastest one, very closely followed by S-MF. The latter requires indeed an additional effort to compute the sensitivities. The S-MILP and L-OPF approaches are significantly slower on the other hand. Note that the difference between the computational time of S-MILP and S-MF is basically the time spent to solve the MILP problem (8-10), which is negligible for small systems with a small number of discrete variables but becomes predominant for larger problems, and may become very high if the MILP problem is badly posed.

2) Minimization of the generation cost: We now compare the performances of various approaches for the OPF problem of generation cost minimization (see Section V-A).

Table VI yields the relative values of the objective obtained by applying the six different approaches. As for the minimum active power losses problem, one can observe that the S-MF approach provides again the best (and near-optimal) solutions. In this case, when feasible, the PRO technique provides very close objective values as the S-MF approach, hence clearly outperforming the RO scheme. Even if initialized by the same continuous relaxation solution as $\mathrm{S}-\mathrm{MF}$ approach, when feasible, the RO approach provides quite poor sub-optimal solution of the MINLP OPF.

One can observe that for the 618-bus system the objective is more sensitive to the values of discrete variables than for the power losses optimization.

By analyzing the results presented in Tables $\mathrm{V}$ and VI one can safely conclude that the S-MF approach is the most appropriate choice for handling discrete variables in an OPF from the viewpoint of objective quality, reliability and 
TABLE VI

MINIMUM GENERATION COST (PU) BY 6 APPROACHES

\begin{tabular}{|c||c|c|c|c|c|c|}
\hline system & c-OPF & RO & PRO & S-MF & S-MILP & L-OPF \\
\hline \hline Nordic32 & 1.0000 & 1.0415 & 1.0007 & 1.0008 & 1.0032 & 1.0253 \\
\hline IEEE300 & 1.0000 & 1.0005 & 1.0003 & 1.0001 & 1.0002 & 1.0004 \\
\hline 618-bus & 1.0000 & 1.0802 & 1.0371 & 1.0348 & 1.0352 & 1.0621 \\
\hline 1203-bus & 1.0000 & fail & fail & 1.0023 & 1.0045 & fail \\
\hline
\end{tabular}

TABLE VII

DISCRETE STEP SIZE OF DISCRETE VARIABLES FOR THREE SETS A, B, C

\begin{tabular}{|c|c|c|c|c|c|c|}
\hline system & \multicolumn{3}{|c|}{ transformer ratio $(\mathrm{pu})$} & \multicolumn{3}{|c|}{ shunt reactive power (MVar) } \\
& $\mathrm{A}$ & $\mathrm{B}$ & $\mathrm{C}$ & $\mathrm{A}$ & $\mathrm{B}$ & $\mathrm{C}$ \\
\hline \hline Nordic32 & 0.005 & 0.010 & 0.020 & $10,15,20$ & $20,30,40$ & $40,60,80$ \\
\hline IEEE300 & 0.005 & 0.010 & 0.020 & $4,10,15,20$ & $8,20,30,40$ & $16,40,60,80$ \\
\hline 618-bus & 0.005 & 0.010 & 0.020 & $10,15,20$ & $20,30,40$ & $40,60,80$ \\
\hline 1203-bus & 0.005 & 0.010 & 0.020 & 15,20 & 30,40 & 60,80 \\
\hline
\end{tabular}

computational time.

\section{E. The effect of using different step sizes for discrete variables}

We now study the impact of using different step sizes for discrete variables on the results obtained with various approaches for both OPF problems: the minimization of power losses and the minimization of the generation cost.

Table VII presents the three sets of step sizes A, B, and C. The set B corresponds to the original step sizes of discrete variables, which have been used in all other simulations presented in the paper, while step sizes of set A (resp. C) have been obtained by dividing (resp. multiplying) by 2 the step sizes of set B.

Table VIII gathers the results obtained with the various approaches when using each of the three sets of step sizes for discrete variables (A, B, C). Note that, as in the experiments presented in Tables $\mathrm{V}$ and VI, the proposed approach S-MF exhibits again better results than the competing approaches. As expected, the larger the step sizes of discrete variables the less good the objective. We have observed that for the minimum power losses problem, the IEEE300 system, and the set C of discrete variables step sizes the MINLP OPF problem is infeasible (i.e. whatever the configuration of discrete variables the feasible domain of continuous variables is empty), due to the large step sizes of discrete controls and tight voltage limits. In this case, after setting up the discrete variables by the S-MF approach, the remaining minimum amount of infeasibility (see Section III-F) is $0.001 \mathrm{pu}$ (in terms of voltage limit violations).

The following sections will investigate different aspects related to the S-MF approach.

\section{F. Robustness of the S-MF approach with respect to weights}

Table IX provides, for different combinations of weights $w_{f}$ and $w_{h}$, the optimal value of both objectives (power losses and generation cost) obtained after setting the values of discrete variables with the S-MF strategy. To fairly assess the impact of these weights we perform only the first iteration in the SMF approach (i.e. solve the continuous relaxation OPF, set the values of discrete variables, and re-optimize the remaining continuous control variables).
TABLE VIII

POWER LOSSES (MW) AND GENERATION COST (PU) FOR THE THREE SETS OF DISCRETE VARIABLES STEP SIZES $(\mathrm{A}, \mathrm{B}, \mathrm{C})$

\begin{tabular}{|c|c|c|c|c|c|c|c|}
\hline system & set & $\mathrm{c}-\mathrm{OPF}$ & $\mathrm{RO}$ & PRO & S-MF & S-MILP & L-OPF \\
\hline \multicolumn{8}{|c|}{ active power losses (MW) at the OPF solution } \\
\hline \multirow{3}{*}{ Nordic32 } & $\overline{\mathrm{A}}$ & $\begin{array}{l}137.79 \\
\end{array}$ & 137.81 & 2137.81 & $\begin{array}{l}137.81 \\
\end{array}$ & 2137.81 & 137.82 \\
\hline & B & 137.79 & 137.92 & 137.92 & 137.82 & 137.81 & 138.02 \\
\hline & $\mathrm{C}$ & 137.79 & 138.66 & 138.54 & 138.09 & 138.02 & 138.57 \\
\hline \multirow{3}{*}{ IEEE300 } & $\mathrm{A}$ & 386.60 & fail & 387.89 & 387.74 & $387.92 *$ & fail \\
\hline & B & 386.60 & fail & fail & 388.06 & $388.49^{*}$ & fail \\
\hline & $\mathrm{C}$ & 386.60 & \multicolumn{5}{|c|}{ problem infeasible } \\
\hline \multirow{3}{*}{ 618-bus } & $\bar{A}$ & 844.25 & 845.38 & 844.96 & 844.65 & 845.14 & 845.26 \\
\hline & B & 844.25 & 846.46 & 846.21 & 845.07 & $845.41^{*}$ & 845.85 \\
\hline & $\mathrm{C}$ & 844.25 & 849.97 & 848.10 & 846.96 & $846.72 *$ & 847.23 \\
\hline \multirow{3}{*}{ 1203-bus } & $\bar{A}$ & 2050.58 & 2052.24 & 2051.89 & 2051.12 & 2051.79 & 2051.85 \\
\hline & B & 2050.58 & 2054.15 & 2053.30 & 2051.67 & $2053.47^{*}$ & 2053.57 \\
\hline & $\mathrm{C}$ & 2050.58 & 2060.20 & 2057.28 & 2053.49 & $2057.38 *$ & 2058.11 \\
\hline \multicolumn{8}{|c|}{ generation cost $(\mathrm{pu})$ at the OPF solution } \\
\hline \multirow{3}{*}{ Nordic32 } & $\mathrm{A}$ & 1.0000 & 1.0002 & 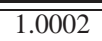 & 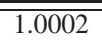 & 1.0002 & 1.0008 \\
\hline & B & 1.0000 & 1.0415 & 1.0007 & 1.0008 & 1.0032 & 1.0253 \\
\hline & $\mathrm{C}$ & 1.0000 & 1.0011 & 1.0011 & 1.0011 & 1.0011 & 1.0032 \\
\hline \multirow{3}{*}{ IEEE300 } & $\mathrm{A}$ & 1.0000 & 1.0004 & 1.0002 & 1.0001 & 1.0002 & 1.0003 \\
\hline & B & 1.0000 & 1.0005 & 1.0003 & 1.0001 & 1.0002 & 1.0004 \\
\hline & $\mathrm{C}$ & 1.0000 & 1.0010 & 1.0009 & 1.0008 & 1.0009 & 1.0010 \\
\hline \multirow{3}{*}{ 618-bus } & $\bar{A}$ & 1.0000 & 1.0309 & 1.0291 & 1.0288 & 1.0294 & 1.0311 \\
\hline & B & 1.0000 & 1.0802 & 1.0371 & 1.0348 & $1.0352 *$ & 1.0621 \\
\hline & $\mathrm{C}$ & 1.0000 & 1.1016 & 1.0521 & 1.0456 & $1.0520 *$ & 1.0721 \\
\hline \multirow{3}{*}{ 1203-bus } & $\bar{A}$ & 1.0000 & 1.0004 & 1.0004 & 1.0006 & 1.0009 & 1.0015 \\
\hline & B & 1.0000 & fail & fail & 1.0023 & $1.0045^{*}$ & fail \\
\hline & $\mathrm{C}$ & 1.0000 & fail & fail & 1.0074 & $1.0078^{*}$ & fail \\
\hline
\end{tabular}

In this table the value of the objective has not been reported for some combinations of weights corresponding to the tests performed on the IEEE300 system. This is because the configurations of discrete variables chosen by the S-MF approach lead to an infeasible continuous OPF problem due to the inability of remaining continuous controls to remove the large voltage limits violation (see section III-F). In such cases, the S-MF approach requires more iterations to convergence.

These results reveal that putting a too strong emphasis on the objective function improvement (e.g. the extreme case where $w_{f}>0$ and $w_{h}=0$, and where $w_{f}=1$ and $w_{h}=100$ ) leads actually to a rather poor value of the objective function ${ }^{3}$. The other extreme case where emphasis is put on removing the limits violation while disregarding the objective function improvement $\left(w_{f}=0\right.$ and $\left.w_{h}>0\right)$ leads to better, however in most cases suboptimal, results.

The combinations of weights $w_{f}=1, w_{h}=100$ and $w_{f}=1, w_{h}=200$ lead to the best results in all cases. Furthermore, for all test systems, both objectives are rather stable over a quite large range of values, i.e. $w_{f}=1$ and $w_{h} \in[50 ; 200]$, which highlights the robustness of the proposed approach. Moreover, both objectives are better than with the round-off technique for an even larger range of weights (see Tables V and VI).

\footnotetext{
${ }^{3}$ At the PF solution, choosing a larger value of $w_{f}$ leads to a configuration of discrete variables which improves the objective but at the expense of larger voltage limits violations (see Table III). This improvement of the objective turns however out to be smaller than the degradation obtained when solving the OPF because the continuous controls must compensate for the larger voltage limit violations.
} 
TABLE IX

THE IMPACT OF THE CHOICE OF WEIGHTS $w_{f}$ AND $w_{h}$ ON THE ACTIVE POWER LOSSES (MW) AND THE GENERATION COST (PU)

\begin{tabular}{|l|l|l|l|l|l|l|l|}
\hline & $w_{f}>0$ & $w_{f}=1$ & $w_{f}=1$ & $w_{f}=1$ & $w_{f}=1$ & $w_{f}=1$ & $w_{f}=0$ \\
& $w_{h}=0$ & $w_{h}=1$ & $w_{h}=10$ & $w_{h}=50$ & $w_{h}=100$ & $w_{h}=200$ & $w_{h}>0$ \\
\hline \hline system & \multicolumn{7}{|c|}{ active power losses (MW) at the OPF solution } \\
\hline Nordic32 & 138.12 & 138.14 & 137.91 & 137.92 & 137.82 & 137.82 & 137.99 \\
\hline IEEE300 & problem infeasible & 388.65 & 388.06 & 388.06 & 388.32 \\
\hline 618-bus & 853.46 & 853.00 & 851.52 & 848.02 & 845.07 & 845.07 & 847.06 \\
\hline $1203-b u s$ & 2066.17 & 2065.75 & 2055.03 & 2051.75 & 2051.67 & 2051.65 & 2052.26 \\
\hline \hline system & \multicolumn{7}{|c|}{ generation cost (pu) at the OPF solution } \\
\hline Nordic32 & 1.0389 & 1.0389 & 1.0027 & 1.0015 & 1.0008 & 1.0010 & 1.0014 \\
\hline IEEE300 & 1.0015 & 1.0008 & 1.0005 & 1.0003 & 1.0003 & 1.0009 & 1.0012 \\
\hline 618-bus & 1.0391 & 1.0391 & 1.0367 & 1.0329 & 1.0348 & 1.0483 & 1.0778 \\
\hline 1203-bus & 1.0158 & 1.0121 & 1.0098 & 1.0078 & 1.0045 & 1.0036 & 1.0022 \\
\hline
\end{tabular}

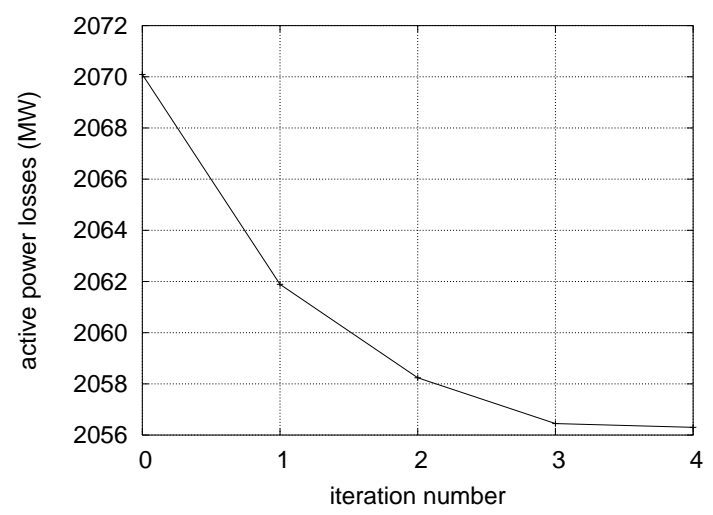

Fig. 3. Evolution of active power losses when starting from a non-optimized base case: results obtained using the 1203-bus system with S-MF

\section{G. Robustness of S-MF approach to its initialization}

We pursue with the 1203-bus system and use $w_{f}=1$ and $w_{h}=100$. We first initialize the discrete variables by means of the round-off approach applied to the continuous OPF relaxation optimum. Then we apply the S-MF approach which indicates that 80 discrete controls (out of 239) should be moved. We move them to their suggested values and solve the OPF with continuous variables only, which leads to power losses of 2053.20 MW, i.e. 0.95 MW less than with the roundoff approach (see Table V). The next iteration decides that no major benefit would be expected by changing the discrete variables values and hence the computation terminates. Thus, when using this initialization, although the losses are improved by $0.95 \mathrm{MW}$ with respect to the round-off approach, they are 1.53 MW higher than when initializing from a continuous OPF relaxation (see Table V). Furthermore, we have observed that even by changing the relative values of weights $w_{f}$ and $w_{h}$, the sub-optimum of 2053.20 MW can not be improved significantly. We conclude that when discrete variables approach very closely their optimal values the information provided by the merit function may be corrupted.

Let us now start from a non-optimized base case of the 1203-bus system and with given values for discrete controls, situation which may correspond to a real-time operating point stemming from the state estimator. In such a case the system operators often look for the best trade-off between the objective improvement and the number of controls moved.
Figure 3 plots the evolution of losses when using this starting point. We observe that the procedure takes 4 iterations to converge and that the improvement is decreasing with the iterations. Also, the final value of losses (2056.30 MW) is slightly larger than with the round-off approach (i.e. 2054.15 MW) and even more suboptimal with respect to S-MF results reported in Table $\mathrm{V}$, corresponding to an initialization from the continuous relaxation OPF version (i.e. 2051.67 MW). An analysis of the converged solution of the procedure actually reveals that the two initializations (continuous relaxation and non-optimized base case) lead to very different settings for most discrete variables. We conclude that depending on the starting point the S-MF method may be "trapped" in a less good sub-optimum, and thus recommend to use whenever possible the continuous relaxation OPF as a starting point.

\section{H. Dealing with infeasible discrete variables configurations}

We now show how the S-MF copes with infeasible situations due to inadequate discrete variable settings, but for which the original MINLP OPF problem is feasible. We illustrate such a case on the IEEE300 system. The active power losses at the relaxed OPF solution are of 386.60 MW (see Table V). Then, after rounding-off the discrete variables, we notice that the subsequent OPF that optimizes continuous variables is infeasible. In contrast, after setting discrete variables by the S-MF approach, the subsequent OPF converges to a solution where the power losses are of $388.06 \mathrm{MW}$, i.e. slightly larger than in the relaxed approach. This illustrates the fact that in some cases the simple logic of the round-off approach may lead to infeasible discrete variable configurations, even if the original MINLP problem is feasible.

Furthermore, to illustrate that the S-MF approach can also provide a solution starting from an infeasible configuration of discrete variables, we first initialize it with the discrete values provided by the round-off approach. Then we solve the OPF which minimizes the problem infeasibility (19-23). The latter provides a feasible solution where two minimum voltage limits have been relaxed with $0.02 \mathrm{pu}$ and $0.01 \mathrm{pu}$, respectively. Next sensitivities are derived at this optimum and discrete variables are moved to new discrete values according to the rules of the heuristic S-MF approach. Finally, by solving the OPF with continuous variables only the problem is indeed found to be feasible and the resulted optimized losses are of 389.79 MW.

\section{CONCLUSIONS}

This paper has proposed and compared three iterative approaches for handling discrete variables in an OPF. The first two approaches rely on analytical sensitivities of the objective and inequality constraints with respect to discrete variables. They set the discrete values for the discrete variables either by solving a MILP problem or by using a simple procedure based on a merit function. The third approach relies on the use of Lagrange multipliers corresponding to the discrete variables bound constraints at the OPF solution.

Extensive numerical results with these approaches on small, medium, and large scale problems have shown that the sensitivity-based approach combined with the merit function 
clearly outperforms the other approaches in terms of: objective function quality, reliability, and computational times. The main features of this approach are summarized in the following way:

- it can pass through infeasible points, using them just as a basis to enable the computation of sensitivities and hence allowing to carry on the procedure;

- it can start from any configuration of discrete variables values (e.g. stemming from the real-time state estimator) or it may be used as a post-processor to other methods, so as to improve the value of the objective function or to find a feasible configuration of the discrete variables. However, much better results in terms of objective quality and computational speed have been obtained when starting from a continuous relaxation OPF solution, the algorithm converging very often in one iteration.

- it restricts at each iteration the movement of each discrete variable to one of its neighboring discrete positions, so as to preserve the validity of sensitivity information;

- a slight drawback of the method is that it depends on the choice of the weights relative to the objective improvement and violated inequality constraints involved in the merit functions. However, the numerical results have shown that the quality of the final optimum is very good for a rather large range of these weights.

Further work should focus on the extension of this approach to cope with discrete variables describing topology changes, which are even more difficult to handle, and on the use of progressive discretization in the S-MF approach.

\section{ACKNOWLEDGMENTS}

We thank RTE (the French transmission system operator) for allowing us to use and publish results with their data.

We also thank the four anonymous reviewers of this paper for their constructive remarks for paper improvement.

This paper presents research results of the Belgian Network DYSCO, funded by the Interuniversity Attraction Poles Programme, initiated by the Belgian State, Science Policy Office. Florin Capitanescu and Louis Wehenkel acknowledge their funding by the FP7 EC project PEGASE. The scientific responsibility rests with the authors.

\section{REFERENCES}

[1] J. Carpentier, "Contribution à l'étude du dispatching économique", Bulletin de la Societé Française d'Electricité, vol. 3, 1962, pp. 431-447.

[2] H.W. Dommel and W.F. Tinney, "Optimal power flow solutions", IEEE Trans. PAS, vol. PAS-87, no. 10, October 1968, pp. 1866-1876.

[3] B. Stott, O. Alsac, and A.J. Monticelli, "Security analysis and optimization" (Invited Paper), IEEE Proc., vol. 75, no. 12, December 1987, pp. 1623-1644.

[4] W.F. Tinney, J.M. Bright, K.D. Demaree, and B.A. Hughes, "Some deficiencies in Optimal Power Flow", IEEE Trans. on Power Syst., vol. 3, 1988, pp. 676-683.

[5] A.D. Papalexopoulos, C.F. Imparato, and F.W. Wu, "Large-Scale Optimal Power Flow: Effects of Initialization, Decoupling \& Discretization", IEEE Trans. on Power Syst., vol. 4, 1989, pp. 748-759.

[6] J.A. Momoh, R.J. Koessler, M.S. Bond, B. Stott, D. Sun, A. Papalexopoulos, and P. Ristanovic, "Challenges to optimal power flow", IEEE Trans. Power Syst. Vol. 12, No. 1, February 1997, pp. 444-455.

[7] K. Karoui, L. Platbrood, H. Crisciu, and R.A. Waltz, "New large-scale security constrained optimal power flow program using a new interiorpoint algorithm", 5-th International Conference on European Electricity Market, May 2008
[8] E. Liu, A.D. Papalexopoulos, and W.F. Tinney, "Discrete Shunt controls in a Newton Optimal Power Flow", IEEE Trans. on Power Syst., vol. 7, 1992, pp. 1519-1528

[9] M. Liu, S.K. Tso, and Y. Cheng, "An Extended Nonlinear Primal-Dual Interior-Point Algorithm for Reactive Power Optimization of Large-Scale Power Systems with Discrete Control Variables", IEEE Trans. on Power Syst., vol. 17, no. 4, 2002, pp. 982-991.

[10] A.G. Bakirtzis and A.P. Meliopoulos, "Incorporation of switching operations in power system corrective control computations", IEEE Trans. on Power Syst., vol. 2, August 1987, pp. 669-676.

[11] S.Y. Lin, Y.C. Ho, and C.H. Lin, "An Ordinal Optimization TheoryBased Algorithm for Solving Optimal Power Flow Problem with Discrete Control Variables", IEEE Trans. on Power Syst., vol. 19, 2004, pp. 276286.

[12] K. Aoki, M. Fan, and A. Nishikori, "Optimal var planning by approximation method for recursive mixed-integer linear programming", IEEE Trans. on Power Syst., vol. 3, November 1988, pp. 1741-1747.

[13] X. Ding, X. Wang, and Y.H. Song, "Interior point cutting plane method for optimal power flow", IMA Journal of management mathematics, vol. 15, no. 4, 2004, pp. 355-368.

[14] L. Liu, X. Wang, X. Ding, H. Chen, "A Robust Approach to Optimal Power Flow With Discrete Variables", IEEE Trans. on Power Syst., vol. 24, no. 3, August 2009, pp. 1182-1190.

[15] A.G. Bakirtzis, P.N. Biskas, C.E. Zoumas, and V. Petridis, "Optimal Power Flow by Enhanced Genetic Algorithm", IEEE Trans. on Power Syst., vol. 17, 2002, pp. 229-236.

[16] L. Chen, H. Suzuki, and K. Katou, "Mean-field theory for optimal power flow", IEEE Trans. on Power Syst., vol. 12, 1997, pp. 1481-1486.

[17] T. Kulworawanichpong and S. Sujitjorn, "Optimal Power Flow using tabu search", IEEE Power Engineering review, 2002, pp. 37-40.

[18] W. Yan, F. Liu, C.Y. Chung, and K.P. Wong, "A Hybrid Genetic Algorithm-Interior Point Method for Optimal Reactive Power Flow", IEEE Trans. on Power Syst., vol. 21, no. 3, August 2006, pp. 1163-1169.

[19] P.R. Gribik, D. Shirmohammadi, S. Hao, and C.L. Thomas, "Optimal Power Flow sensitivity analysis", IEEE Trans. on Power Syst., vol. 5, no. 3, August 1990, pp. 969-978.

[20] GLPK (GNU Linear Programming Kit) solver, available online at: http://www.gnu.org/software/glpk/.

[21] OPF data and numerical results of 60-bus and IEEE300-bus systems, available online at http://www.montefiore.ulg.ac.be/ capitane/, 2009

[22] CIGRE Task Force 38.02.08, "Long-Term Dynamics, Phase II", 1995.

[23] IEEE300 bus system, available online at http://www.ee.washington.edu, 1996.

[24] F. Capitanescu, M. Glavic, D. Ernst, and L. Wehenkel, "Interior-point based algorithms for the solution of optimal power flow problems", Electric Power Syst. Research, vol. 77, no. 5-6, April 2007, pp. 508-517.

Florin Capitanescu graduated in Electrical Power Engineering from the University "Politehnica" of Bucharest in 1997. He obtained the Ph.D. degree from the University of Liège in 2003. His main research interests include optimization methods and voltage stability.

Louis Wehenkel (M'93) graduated in Electrical Engineering (Electronics) in 1986 and received the Ph.D. degree in 1990, both from the University of Liège, where he is full Professor of Electrical Engineering and Computer Science. His research interests lie in the fields of stochastic methods for systems and modeling, optimization, machine learning and data mining, with applications in complex systems, in particular large scale power systems planning, operation and control, industrial process control, bioinformatics and computer vision. 